\title{
Interrupção e Retomada da Vida Sexual após o Câncer de Mama
}

\author{
Daniela Barsotti Santos ${ }^{1}$ \\ Univesidade Federal do Rio Grande \\ Manoel Antônio dos Santos \\ Vanessa Monteiro Cesnik-Geest \\ Elisabeth Meloni Vieira \\ Universidade de São Paulo
}

\begin{abstract}
RESUMO - Investigou-se a interrupção/retomada da vida sexual após o tratamento do câncer de mama e sua relação com a satisfação e outros aspectos valorizados em um relacionamento. Foram empregados métodos mistos de pesquisa com a análise dos dados de uma survey realizada com 139 mulheres e dados provenientes de entrevistas com roteiro semiestruturado realizadas com 24 participantes. Um percentual expressivo de mulheres (66\%) sexualmente ativas interrompeu as atividades sexuais durante o tratamento. Observou-se que a interrupção e retomada da vida sexual relacionou-se às concepções pessoais de sexualidade, influenciadas pelas relações de gênero e pela qualidade do relacionamento amoroso. A identificação de necessidades relacionadas à intimidade sexual pelo profissional de saúde pode contribuir para a assistência apropriada no processo de reabilitação psicossocial da mulher.
\end{abstract}

Palavras-chave: neoplasias mamárias, sexualidade, intercurso sexual, conflito conjugal, dinâmica de casal

\section{Interruption and Re-Start of Sex Life after Breast Cancer}

\begin{abstract}
Interruption and the re-start of sex life after breast cancer treatment as well as satisfaction and other valued aspects of the relationship were investigated. Mixed methods of data analysis were used including the results of a survey with 139 women and qualitative data of 24 interviews using a semi-structured guideline. An expressive proportion of women $(66 \%)$ sexually active interrupted their sexual activities during the treatment. The results indicated that interruption and restart of sex life is related to personal conceptions of sexuality, and is influenced by the concept of gender and the quality of the relationship. The identification of needs related to the sexual intimacy by health professional can contribute to appropriate care of women in their process of psychosocial rehabilitation.
\end{abstract}

Keywords: breast neoplasms, sexuality, sexual intercourse, intimacy, marital conflict, couple dynamics

Atualmente, cada vez mais se reconhece que as alterações no bem-estar sexual podem ser uma das dimensões mais problemáticas da vida após o câncer de mama, com impacto duradouro que pode se estender por muitos anos após um tratamento bem-sucedido (Silva \& Santos, 2008), mas muitas vezes associado a graves danos físicos e emocionais, decorrentes de efeitos colaterais e da elevada toxicidade do tratamento (Ussher, Perz, \& Gilbert, 2013). Embora alguns estudos relatem altos níveis de qualidade de vida no início do período pós-tratamento do câncer de mama (Ganz et al., 2002), sequelas físicas e psicológicas podem persistir por um tempo relativamente prolongado (Ambrósio \& Santos, 2011, 2015; Peres \& Santos, 2007, 2009; Silva \& Santos, 2010; Ussher et al., 2013), dentre as quais aparecem com destaque as dificuldades sexuais (Brédart et al., 2011; Cesnik \& Santos, 2012a, 2012b; Cesnik et al., 2013; Klaeson, Sandell, \& Bertero, 2011; Santos \& Vieira, 2011; Santos, Ford, Santos, \& Vieira, 2014; Sawin, 2012; Ussher, Perz, \& Gilbert, 2012; Vieira, Santos, Santos, \& Giami, 2014).

Muitas vezes o foco principal dos estudos é colocado nas mudanças corporais, o que acaba obscurecendo a

1 Endereço para correspondência: Departamento de Medicina Social, Faculdade de Medicina de Ribeirão Preto, Universidade de São Paulo. Av. Bandeirantes, 3900, Monte Alegre, Ribeirão Preto, SP, Brasil. CEP: 14.049-9000,E-mail: danibarsotti@yahoo.com.br influência do ambiente social e das construções relacionais da sexualidade e da doença (Scorsolini-Comin, Santos, \& Souza, 2009). Além desses aspectos pouco enfatizados pela literatura, também são negligenciadas as formas como significados atribuídos à vida afetivo-sexual são negociados pelas pessoas engajadas em seus relacionamentos (Ussher et al., 2012).

Pesquisas evidenciam que a maioria das mulheres recupera sua atividade sexual após o tratamento do câncer de mama (Barni \& Mondin, 1997; Fobair et al., 2006; Ganz et al., 1999; Meyerowitz, Desmond, Rowland, Wyatt, \& Ganz, 1999; Takahashi et al., 2008). No entanto, tais mulheres relatam mais preocupações sobre sua sexualidade do que as mulheres da população geral (Rowland et al., 2009; Speer et al., 2005). A literatura mostra que, quando comparadas às mulheres saudáveis na mesma idade, as mulheres com câncer de mama experimentam níveis mais baixos de satisfação sexual e têm mais dificuldade em manter sua vida sexual (Speer et al., 2005).

Estudo realizado com o objetivo de avaliar a prevalência e os fatores associados à atividade sexual, problemas sexuais ou satisfação sexual em mulheres francesas sobreviventes ao câncer de mama em estágio inicial (Brédart et al., 2011) investigou 453 mulheres, no período pós-tratamento (seis meses a cinco anos), com idades entre 18 e 70 anos, que 
foram selecionadas aleatoriamente de uma lista de consulta com 850 mulheres que aceitaram participar do estudo. As participantes eram mais jovens, estavam com maior frequência na pré-menopausa quando foram diagnosticadas e tinham diagnóstico mais recente do que as mulheres do grupo de comparação, obtidas de uma amostra representativa da população francesa. A prevalência de problemas sexuais foi significativamente maior em comparação com dados ajustados a partir da amostra representativa. A interrupção da atividade sexual e insatisfação sexual foram associadas à presença de um sentimento de distanciamento emocional no casal ou ao medo, sentido pelo parceiro, de ter relação sexual, bem como menor nível de funcionamento emocional, imagem corporal empobrecida ou presença de comorbidades.

Em estudo realizado na Austrália, Ussher et al. (2012) examinaram as alterações em relação à sexualidade e nos relacionamentos íntimos em pessoas de ambos os sexos acometidas pelo câncer de mama. Foram utilizados métodos mistos de pesquisa e a análise foi guiada por uma "perspectiva material-discursivo-intrapsíquica". Foi feito um levantamento on-line com 47 questões quantitativas e qualitativas. A amostra abarcou 1965 indivíduos com câncer mamário, dos quais $98 \%$ eram mulheres, com média de idade de 54 anos. Os resultados mostraram declínio na frequência, resposta e satisfação sexual, atribuídos pelos participantes a fatores como fadiga e dor, sofrimento (distress) psicológico e prejuízos na imagem corporal, além de alterações decorrentes da menopausa induzida pelo tratamento, como secura vaginal, fogachos e ganho de peso. As preocupações dominantes foram as consequências emocionais, mudanças físicas, sentimento de não ter mais atratividade sexual ou falta de feminilidade, reconciliação do self frente às mudanças e impacto sobre o parceiro ou no relacionamento afetivo.

Apoiando-se nesse panorama da literatura, pode-se afirmar que os problemas sexuais que aparecem depois da cirurgia de retirada do nódulo mamário e da quimioterapia têm sido frequentemente relatados (Cesnik et al., 2013; Fobair et al., 2006; Janz et al., 2005; Santos, Santos, \& Vieira, 2014; Vieira et al., 2014). Todavia, são poucos os estudos brasileiros sobre o impacto observado na atividade sexual em sobreviventes ao câncer de mama. Considerando essa lacuna do conhecimento, o presente estudo teve por objetivos: (1) investigar a interrupção e retomada da vida sexual em decorrência do tratamento oncológico em mulheres com câncer de mama; (2) explorar a satisfação com o relacionamento amoroso, assim como a percepção de alguns aspectos que são valorizados em um relacionamento e sua relação com a interrupção e retomada da atividade sexual após o câncer de mama.

\section{Método}

Foram utilizados métodos mistos de pesquisa em um desenho de estudo do tipo convergente, no qual os dados quantitativos foram extraídos de uma survey e os dados qualitativos obtidos por meio de entrevistas guiadas por roteiro semiestruturado. Segundo esse tipo de desenho de estudo, os dados são analisados separadamente, para que se possa posteriormente uni-los e, assim, obter maior clarificação sobre o fenômeno estudado (Creswell \& Clark, 2011).

Métodos mistos de pesquisa envolvem a triangulação de informações obtidas a partir de diferentes fontes de dados (Lund, 2012). Os dados dos componentes quantitativo e qualitativo foram triangulados a fim de examinar os aspectos que podem ser complementares, convergentes ou divergentes (Denzin, 2009). Optou-se por delimitar a triangulação dos dados a partir das variáveis utilizadas no componente quantitativo (Creswell \& Clark, 2011).

\section{Componente Quantitativo}

Os dados quantitativos foram coletados entre setembro de 2010 e janeiro de 2011, por meio de entrevistas realizadas com 139 mulheres, que haviam sido diagnosticadas com câncer de mama havia pelo menos seis meses, no período entre 2006 e 2010. Todas as participantes eram frequentadoras de um serviço de reabilitação para mulheres mastectomizadas, oferecido pela Escola de Enfermagem de Ribeirão Preto da Universidade de São Paulo. Como instrumento de coleta de dados, foi utilizado um questionário composto por 110 perguntas, aplicadas face a face por entrevistadoras treinadas.

Calculou-se o tamanho amostral para uma população finita considerando a prevalência de $50 \%$ em relação à atividade sexual no último mês, erro de $5 \%$ e nível de significância de 5\%, resultando em ao menos 132 indivíduos. A partir de uma lista com os nomes de todas as mulheres inscritas no serviço foram sorteadas 184 mulheres, contatadas por telefone ou pessoalmente. Desse total, 14 já tinham falecido, 17 se recusaram a colaborar com o estudo e 14 não foram encontradas. As entrevistas foram realizadas nas residências, em todos os dias da semana, inclusive aos finais de semana e feriados, em qualquer período do dia, dependendo da disponibilidade da entrevistada. Quando não pôde ser completado o contato telefônico, foram realizadas até três visitas ao endereço indicado nos prontuários. Caso a mulher sorteada não fosse encontrada, era excluída do estudo e substituída por outra.

Para responder aos objetivos deste estudo foram analisadas as seguintes variáveis do componente quantitativo: (1) Interrupção e retomada da vida sexual: foi perguntado se a respondente havia parado de ter relação sexual durante os tratamentos do câncer de mama e, em caso afirmativo, quanto tempo depois ela havia retomado; (2) Motivo ou iniciativa da retomada da vida sexual; (3) Satisfação com o relacionamento amoroso, avaliada por quatro afirmações que descreviam um gradiente de variação entre "este relacionamento não preenche minhas necessidades afetivas" até "preenche totalmente minhas necessidades afetivas"; (4) A percepção de outros aspectos, tais como fidelidade conjugal, satisfação com a renda familiar, admiração pelo parceiro e crenças religiosas, foram avaliados com o propósito de conhecer se são valores considerados importantes ou não em um relacionamento conjugal.

Para análise dos dados quantitativos utilizou-se estatística descritiva e testes de associação entre as variáveis, como o qui-quadrado de Pearson e o teste exato de Fischer. O nível de significância adotado foi $p \leq 0,05$. 


\section{Componente Qualitativo}

Os dados qualitativos foram obtidos por meio de entrevistas, aplicadas mediante roteiro semiestruturado, administrado a 24 mulheres diagnosticadas com câncer de mama. $\mathrm{O}$ número total de participantes foi definido pela saturação dos temas relacionados ao objetivo do estudo. Os dados foram audiogravados e transcritos integralmente, constituindo o corpus de análise. Os dados sociodemográficos e as informações sobre o diagnóstico e tratamento foram coletados com as participantes e por meio de consulta aos prontuários.

Para os dados qualitativos utilizou-se análise temática de conteúdo, definindo-se categorias a priori. O material foi codificado duas vezes para a certificação do recorte dos extratos relevantes para cada categoria de análise, com o auxílio do programa NVivo9.

Os dados foram discutidos com base na literatura da área $\mathrm{e}$ no referencial teórico do gênero. Há uma literatura crescente sobre gênero e determinantes de saúde. Para Ballantyne (1999), a categoria gênero medeia os diferentes modos como mulheres e homens experimentam os vínculos amorosos e a forma de se integrarem socialmente. Consistente com esse pressuposto teórico, a posição assumida por este estudo é a de que o gênero medeia a influência desses vínculos sobre a saúde da mulher. Para as mulheres, esses aspectos - a forma de se conduzir na vida amorosa e sua percepção do processo saúde-doença - são intrinsecamente ligados. Por isso, para elas o relacionamento conjugal pode ter um papel paradoxal: o casamento pode, por um lado, incrementar suas oportunidades de receber apoio social, ao passo que a manutenção das desigualdades de gênero na relação pode potencializar sua vulnerabilidade, comprometendo suas condições de saúde. Para a discussão dos dados qualitativos também foi utilizada a concepção teórica proposta por Giddens (2003) sobre os relacionamentos amorosos e a transformação da intimidade na sociedade ocidental.

A pesquisa foi conduzida dentro dos padrões exigidos pela Declaração de Helsinque e aprovada pelo Comitê de Ética em Pesquisa da Escola de Enfermagem de Ribeirão Preto, da Universidade de São Paulo, parecer 193/2009. Todas as entrevistadas assinaram o Termo de Consentimento Livre e Esclarecido.

\section{Resultados}

Os dados referentes às características sociodemográficas da amostra, à história reprodutiva e sexual das participantes, bem como aos diagnósticos e tratamentos a que foram submetidas podem ser encontrados em publicação anterior (Vieira et al., 2013).

\section{Interrupção da Vida Sexual}

Observou-se que, entre as 139 participantes, 94 (67,5\%) relataram ter vida sexual ativa na ocasião do diagnóstico de câncer de mama. Destas, 62 (66\%) mulheres declararam que interromperam as relações sexuais em algum momento do tratamento.

As mulheres entrevistadas no componente qualitativo do estudo relataram alguns motivos para a interrupção da vida sexual, como angústia e estresse suscitados pelo diagnóstico do câncer de mama, a recuperação da cirurgia mamária e a indisposição física provocada pela quimioterapia.

$E$ [entrevistadora]: Nesse tempo desde que você fez a cirurgia, vocês chegaram a parar de ter relação sexual?

Amanda: Para mim, o início do tratamento foi um caos. Porque, eu não passei mal com a quimioterapia, mas foi como se passasse um rolo compressor. A gente fica com o corpo moído. Ai cai o cabelo, você se sente assim... Apesar de que meu marido, nossa, me acompanhou em tudo (Amanda, 51 anos, casada).

Observou-se que 32 (34\%) mulheres não interromperam a vida sexual durante os tratamentos. Em algumas falas das entrevistadas do componente qualitativo, duas explicações contrastantes surgiram para fundamentar a motivação para a manutenção da vida sexual durante o período pós-operatório. Para algumas participantes a continuidade das relações sexuais deveu-se ao bom relacionamento afetivo-sexual anterior com o parceiro, sendo a relação sexual entendida como uma expressão do amor pelo mesmo. $\mathrm{O}$ intercurso sexual como fonte de obtenção de prazer sexual, por parte da mulher, é um aspecto praticamente ausente nas justificativas apresentadas referentes à vida sexual do casal.

Já outras participantes referiram-se às relações sexuais como um dever, uma obrigação conjugal da esposa, inclusive como estratégia para evitar uma possível traição do marido, caso a mulher se recusasse a "servi-lo" sexualmente.

Ana: [...] eu sirvo ele toda hora que ele quer [...]. Nunca falei não, nunca. [...] A gente casou, a gente tem que servir eles na hora que eles querem.

Entrevistadora: Mas o que acontece se a mulher falar não?

Ana: Ah, eu tenho a impressão que vai atrair o que não presta. Ele vai procurar na rua (Ana, 57 anos, casada).

\section{Retomada da Vida Sexual}

No momento da entrevista $55(39,6 \%)$ mulheres haviam retomado a vida sexual, após uma média de sete meses e seis dias de interrupção (mediana de cinco meses, desvio-padrão 1,21). Das 55 mulheres que retomaram as relações sexuais, $27(49 \%)$ reiniciaram por iniciativa própria, enquanto que $18(32,6 \%)$ participantes relataram que voltaram a manter relações sexuais para agradar o parceiro ou por insistência dele. Ainda, $10(18,2 \%)$ mulheres afirmaram ter retomado a vida sexual por iniciativa do casal ou por ter arrumado um novo parceiro. Dessas, cinco mulheres já não estavam mais envolvidas em um relacionamento afetivo.

\section{Satisfação com o Relacionamento Amoroso}

Do total de mulheres que responderam ao questionário, $85(61 \%)$ declararam que estavam envolvidas em um relacionamento amoroso no momento da entrevista. Em relação à satisfação com o relacionamento amoroso, a maioria das participantes estava plenamente satisfeita ou parcialmente 
satisfeita com o relacionamento afetivo, enquanto que uma parcela menor concordava que o relacionamento preenchia muito pouco ou não preenchia de forma alguma suas necessidades afetivas (Tabela 1).

Tabela 1. Frequência Absoluta e Percentual das Participantes de Acordo com a Satisfação com o Relacionamento Amoroso em Relação ao Preenchimento de Necessidades Afetivas

\begin{tabular}{lrc}
\hline \multicolumn{1}{c}{ Satisfação com o relacionamento amoroso } & f & \% \\
\hline Preenche totalmente as necessidades afetivas & 42 & 49,3 \\
Preenche em parte & 23 & 27,1 \\
Preenche muito pouco & 10 & 11,8 \\
Não preenche de jeito algum & 10 & 11,8 \\
Total & 85 & 100 \\
\hline
\end{tabular}

No componente qualitativo da pesquisa, algumas entrevistadas atribuíram ao câncer de mama mudanças tanto positivas quanto negativas em seu relacionamento afetivo, sendo a doença percebida como um evento vital que fortaleceu ou, por outro lado, desintegrou uma relação amorosa que já estava desgastada.

Luciana: [...] não que ele não gostasse de mim antes, mas parece que ele passou a demonstrar mais, sabe? [depois do câncer de mama] Assim, ele disse para mim que no dia em que ele foi me ver no hospital, que eu tinha operado, que ele entrou pela porta e ele falou, ele até chorou falando isso para mim, naquela hora ele percebeu o quanto que eu era importante para ele. E que ele percebeu que me amava mesmo. Então, isso foi muito compensador (Luciana, 42 anos, divorciada, namorando atualmente).

Juçara: Sou casada e me arrependi de ter casado. [...] Estou pensando no divórcio. [...] Não tenho mais paciência não, entendeu? Não dá. Depois que eu tive esse câncer, parece que ficou pior, sabe, essa zoação. Eu gosto de ser sozinha. De ficar sozinha no meu canto. Eu só luto pelo câncer porque eu tenho uma filha de seis anos, senão, eu não lutaria contra ele (Juçara, 36 anos, casada).

Uma parte das mulheres que definiu seu relacionamento como bom apontou como qualidades que admiravam no parceiro: compreensão, apoio, afeto e respeito. Algumas relataram que seus parceiros demonstraram sentimentos de amor, de temor por uma eventual perda da parceira e de pesar pelo sofrimento vivenciado durante os tratamentos. Além disso, ofereceram suporte emocional e as acompanharam nas consultas médicas, tratamentos e exames. Alguns parceiros foram os principais cuidadores, encarregando-se de realizar a troca de curativos, cuidados com dreno, auxílio na higiene pessoal e vestimenta da mulher, além de algumas vezes terem substituído as mulheres na realização dos afazeres domésticos e no cuidado dos filhos.

Uma das entrevistadas afirmou que sempre manteve um bom relacionamento com seu marido, mas que ficou surpresa e comovida com o forte apoio recebido durante os tratamentos para o câncer de mama.

Rita: Cuidadoso, atencioso, contava para todo mundo na esperança que: "Ai mulher, fulana teve o mesmo, mas sarou! A outra teve, mulher". Ele ficava pesquisando. Nunca me deixou sozinha. Quando eu fiz o dreno, ele cuidava. [...] Me deu banho, rapou minha cabeça. [...] Ele foi muito amigo. Um homem diferente, esse homem eu nem conhecia ele [voz trêmula]. E nem conheço, porque depois que passou a fase... Sarou, eles voltam ao normal. [...] Agora é na casca dura mesmo. Mas naquela época ele [...] caiu ali para me socorrer, sabe?" (Rita, 54 anos, casada).

Relacionamentos considerados conturbados ou difíceis, desde o seu início, tiveram piora, em maior ou menor grau, atribuída ao acometimento pelo câncer de mama. As mulheres relataram outros fatores de desgaste do relacionamento afetivo, anteriores à doença:

Juçara: Meu marido. Ele é bom, não bebe, não fuma. Eu falo para ele: "É dificil". Tem hora que a gente leva cada patada. $E$ estranho. Não precisa ficar adulando a gente, mas uma patada que dá nessas horas a gente fica muito frágil. Igualzinho eu, quando tomo a quimio, eu fico no chão, fico arrasada. Eu fico muito sensível, entendeu? Não dá. Ele pensa de um jeito, eu penso de outro (Juçara, 36 anos, casada).

Foi encontrada associação estatisticamente significante $(p=0,012)$ entre insatisfação ou satisfação parcial com o relacionamento afetivo e a retomada da vida sexual para agradar o parceiro ou por insistência dele. Das 50 mulheres que estavam em um relacionamento e haviam retomado a vida sexual, $28(56 \%)$ referiram que foi por iniciativa delas ou de ambos, enquanto 17 (34\%) mencionam que foi para agradar o parceiro ou por insistência dele. Enquanto que apenas $23,5 \%$ das que referiram ter satisfação plena com o relacionamento retomaram as atividades sexuais para agradar o parceiro, $76,5 \%$ das que estavam insatisfeitas com a vida sexual o fizeram para agradar o parceiro (Tabela 2).

Essa questão também foi analisada nas entrevistas do componente qualitativo. Os relatos sugerem que cabe à mulher regular e manter o interesse sexual do marido nos limites do espaço privado, de modo a evitar que ele busque reconfortar-se nos braços de outra mulher, disposta

Tabela 2. Distribuição das Participantes de Acordo com o Motivo Principal da Retomada da Vida Sexual em Função da Satisfação com o Relacionamento com o Parceiro*

Motivo principal da retomada da vida sexual

Satisfação com o relacionamento estabelecido com o parceiro, em função do preenchimento das necessidades afetivas

\begin{tabular}{lccc}
\cline { 2 - 4 } & Preenche totalmente & $\begin{array}{c}\text { Preenche em parte, } \\
\text { pouco ou não preenche }\end{array}$ & Total \\
\cline { 2 - 4 } Por iniciativa própria ou de ambos & $18(64,3 \%)$ & $10(35,6 \%)$ & $28(100 \%)$ \\
Para agradar o parceiro ou por insistência dele & $4(23,5 \%)$ & $13(76,5 \%)$ & $17(100 \%)$ \\
Total & $22(100 \%)$ & $23(100 \%)$ & $45(100 \%)$ \\
\hline
\end{tabular}

Notas. ${ }^{*}$ Teste Qui-quadrado de Pearson $=8,7845, \mathrm{p}=0,012, *$ Teste de Fisher $=0,009$ 
a seduzir e a lhe roubar o esposo. Ceder e recomeçar a vida sexual por insistência do parceiro ou para agradá-lo está relacionado a uma concepção de sexualidade pautada nas relações tradicionais de gênero, em que o relacionamento afetivo estável traria reconhecimento social e respeitabilidade à mulher. Além disso, há uma dupla moral sexual, que nega o desejo sexual feminino e naturaliza o desejo sexual masculino. Nessa acepção, é fundamental que a mulher esteja sempre atraente sexualmente e disposta a manter relações sexuais para manter seu parceiro satisfeito, uma vez que sempre existiria alguma outra mulher disposta a fazer mais sexo do que ela. Todavia, esse imperativo de se manter permanentemente em estado de prontidão para gratificar as necessidades sexuais do parceiro, na tentativa de aplacar sua insatisfação, torna-se ainda mais difícil de ser mantido quando a mulher está enfrentando os desconfortos físicos e psíquicos, bem como as alterações corporais decorrentes do tratamento oncológico. Isso pode gerar conflitos e incrementar ainda mais o sofrimento, gerando distress psicológico.

Entre as entrevistadas do componente qualitativo, houve relatos sobre o temor do desprezo e possível abandono por parte do parceiro, devido às mudanças corporais que os tratamentos do câncer de mama acarretam. Essas alterações afetam a atratividade sexual da mulher e o sentimento de ser desejada, impactando a intimidade conjugal.

Paula: Eu já conversei com pessoas [...] senhoras novas [...] com menos de 50 anos [riso], ainda mais moça e... bonita, vistosa. Sabe o quê que ela me disse? Que o marido dela quando... soube que ela ia tirar o seio, ele pegou e desprezou ela [...] falou que ela tava tudo podre por dentro (Paula, 60 anos, casada).

É interessante observar que, apesar de mencionarem o medo do abandono pelo parceiro, nenhuma das mulheres afirmou ter sido deixada pelo companheiro em razão do adoecimento. Ademais, no componente quantitativo, foram identificadas cinco $(9 \%)$ das 55 mulheres que retomaram à vida sexual após os tratamentos em razão do início de um novo relacionamento amoroso (Tabela 2). Outro achado relevante é que também foi identificada, em algumas mulheres, uma concepção de sexualidade que valoriza o bemestar e o prazer sexual feminino como conteúdos inerentes à vivência da intimidade conjugal. Mas essas expressões da sexualidade aparecem estreitamente associadas ao sentimento da mulher pelo parceiro:

Entrevistadora: O que é sexualidade pra você?

Luciana: [...] acho que sexualidade está em tudo, na pele, no olhar, no toque. [...] Você não vai ali transar com seu namorado pelo simples fato de que seu corpo está querendo! Mas é um conjunto de coisas. [...] Você vê que você tem um sentimento por ele (Luciana, 42 anos, divorciada, namora atualmente).
Entre as mulheres que relataram que houve interrupção da vida sexual, uma parcela expressiva $(43,2 \%)$ mantém, atualmente, relações sexuais com uma frequência de três a quatro vezes ao mês (Tabela 3 ). Entre aquelas que não interromperam a vida sexual durante os tratamentos, 57,7\% referiram ter relações acima de cinco vezes por semana.

\section{Valores Considerados Importantes em um Relacionamento}

Compreender os significados atribuídos ao relacionamento afetivo-sexual pelas participantes deste estudo é importante para um entendimento mais abrangente do papel da sexualidade na experiência do câncer de mama. Foram coletadas, com as 139 participantes do componente quantitativo, as opiniões sobre a relevância de determinados aspectos inerentes a um relacionamento amoroso (Tabela 4). Todos os atributos apresentados receberam mais respostas concentradas entre as alternativas "muito importante" e "importante", sendo que "respeito e admiração pelo parceiro" (100\%) e "fidelidade" $(98,5 \%)$ foram os aspectos mais valorizados pelas mulheres, enquanto que o "compartilhamento de crenças religiosas" (81\%) e "ter gostos e interesses em comum" $(75,2 \%)$ foram as assertivas comparativamente menos apreciadas, embora ainda bastante desejáveis em um relacionamento.

A maioria das participantes descreveu o casamento como um ideal feminino a ser alcançado, sob um panorama de relações tradicionais de gênero. Nesse contexto, o amor romântico aparece como sentimento fundamental para alicerçar o relacionamento afetivo-sexual do casal.

Paula: Ai, quando ele olhou pra mim e eu olhei pra ele, parece que tava escrito. [...] Ai ele falou assim, que ele olhou pra mim e parecia que eu era a mais bonita de todas [riso]. Ai eu disse pra ele, né: "Só que pra você viver comigo, eu fiz essa opção de casar de noiva" (Paula, 60 anos, casada).

No entanto, algumas falas ilustram características de um amor confluente, isto é, um tipo de união baseada na reciprocidade e em relações mais igualitárias entre os sexos.

Vivian: [...] Mas, assim, o relacionamento não é só sexual. Você tem que ter uma vida. Uma vida assim, de igual para igual, não competitividade entre o casal. Mas um companheirismo junto, na vida a dois, da família (Vivian, 51 anos, casada).

\section{Discussão}

Os resultados evidenciaram que um percentual expressivo de mulheres $(66 \%)$, que eram sexualmente ativas por ocasião do diagnóstico de câncer de mama, apresentou

Tabela 3. Interrupção ou Não da Vida Sexual Comparada com a Frequência Sexual Atual*

\begin{tabular}{ccccc}
\hline \multirow{2}{*}{$\begin{array}{c}\text { Interrupção da vida } \\
\text { sexual }\end{array}$} & \multicolumn{4}{c}{ Frequência de relações sexuais } \\
\cline { 2 - 5 } & $\mathbf{1}$ a 2 vezes ao mês & 3 a 4 vezes ao mês & $\mathbf{5}$ a 45 vezes ao mês & Total \\
\hline Sim & $12(27,3 \%)$ & $19(43,2 \%)$ & $13(29,5 \%)$ & $44(100 \%)$ \\
Não & $6(23,1 \%)$ & $5(19,1 \%)$ & $15(57,7 \%)$ & $26(100 \%)$ \\
Total & $18(25,7 \%)$ & $24(34,3 \%)$ & $28(40,0 \%)$ & $70(100 \%)$ \\
\hline Notas. *Teste Qui-quadrado de Pearson $=6,0832, p=0,048 ; *$ Teste de Fisher $=0,047$ & &
\end{tabular}


Tabela 4. Valores Considerados Relevantes em um Relacionamento Amoroso

\begin{tabular}{|c|c|c|c|c|c|}
\hline $\begin{array}{l}\text { Valores considerados relevantes em um } \\
\text { relacionamento }\end{array}$ & $\begin{array}{c}\text { Muito } \\
\text { importante }\end{array}$ & Importante & $\begin{array}{l}\text { Não é muito } \\
\text { importante }\end{array}$ & $\begin{array}{c}\text { Não é } \\
\text { importante }\end{array}$ & Total \\
\hline Importância da fidelidade em um relacionamento* & $112(81,2 \%)$ & $24(17,4 \%)$ & $2(1,4 \%)$ & - & $138(100 \%)$ \\
\hline Importância da renda adequada em um relacionamento* & $45(32,5 \%)$ & $77(55,8 \%)$ & $14(10 \%)$ & $2(1,4 \%)$ & $138(100 \%)$ \\
\hline $\begin{array}{l}\text { Importância do respeito e admiração pelo parceiro em um } \\
\text { relacionamento }\end{array}$ & $119(85,5 \%)$ & $20(14,4 \%)$ & - & - & $139(100 \%)$ \\
\hline $\begin{array}{l}\text { Importância do compartilhamento de crenças religiosas } \\
\text { pelo casal em um relacionamento* }\end{array}$ & $58(42,2 \%)$ & $53(38,7 \%)$ & $17(12,3 \%)$ & $9(6,6 \%)$ & $137(100 \%)$ \\
\hline $\begin{array}{l}\text { Importância de um relacionamento sexual feliz em um } \\
\text { relacionamento* }\end{array}$ & $66(48,2 \%)$ & $61(44,4 \%)$ & $8(5,7 \%)$ & $2(1,5 \%)$ & $137(100 \%)$ \\
\hline $\begin{array}{l}\text { Importância da divisão de tarefas domésticas em um } \\
\text { relacionamento }\end{array}$ & $44(31,6 \%)$ & $71(51,1 \%)$ & $17(12,1 \%)$ & $7(5 \%)$ & $139(100 \%)$ \\
\hline Importância de ter filhos em um relacionamento* & $67(48,5 \%)$ & $51(37 \%)$ & $12(8,7 \%)$ & $8(5,8 \%)$ & $138(100 \%)$ \\
\hline $\begin{array}{l}\text { Importância de ter gostos e interesses comuns em um } \\
\text { relacionamento* }\end{array}$ & $30(21,9 \%)$ & $73(53,3 \%)$ & $24(17,4 \%)$ & $10(7,3 \%)$ & $137(100 \%)$ \\
\hline
\end{tabular}

Nota. *Não foram incluídos os dados perdidos

interrupção das relações sexuais durante o tratamento. Os dados obtidos apoiam achados derivados de estudos anteriores, que referem alterações significativas no bemestar sexual da mulher após o diagnóstico e tratamento do câncer de mama (Brédart et al., 2011; Klaeson et al., 2011; Sawin, 2012; Ussher et al., 2012). Neste último estudo, foi encontrado declínio na frequência, resposta e satisfação sexual, atribuídos pelas mulheres a fatores como fadiga, dor, distress psicológico, prejuízos na imagem corporal e alterações físicas decorrentes da menopausa precoce induzida pelo tratamento. Segundo Klaeson et al. (2011), o tratamento com drogas antineoplásicas leva as mulheres a vivenciarem seu corpo de uma maneira totalmente nova e desconhecida. Essas experiências corporais potencializam a sensação de ter se tornado uma estranha (outsider), de não ser mais a mulher que era antes do adoecimento, e esse déficit no plano identitário afeta profundamente sua sexualidade e feminilidade. A propósito, Peres e Santos (2012) avaliaram a personalidade de mulheres com câncer de mama por meio de técnicas projetivas e concluíram que nelas o corpo, em vez de ser um meio de satisfação pulsional, passa a ser investido também como veículo de expressão do sofrimento, em especial daquele que não encontra via de manifestação em termos psíquicos.

A retomada da vida sexual demandou, em média, sete meses, o que pode ser considerado um intervalo prolongado de interrupção. Esse dado sugere dificuldades de adaptação ao novo contexto de vida, iniciado com o diagnóstico e o tratamento para o câncer de mama. Por outro lado, algumas mulheres se mostram aliviadas com a possibilidade de cessar a vida sexual (Sawin, 2012). Embora cerca da metade (49\%) das mulheres tenha reiniciado a vida sexual por iniciativa própria, entre os motivos da retomada destaca-se o desejo de agradar o parceiro, observado em uma parcela importante (32,6\% das participantes). Novamente percebe-se a subordinação a uma prescrição de gênero, que designa às mulheres o "dever" de preservar o casamento, assegurando a satisfação sexual do parceiro, mesmo que isso represente contrariar seus próprios desejos e comprometa seu bem-estar físico e emocional. Nesse contexto, o tratamento oncológico e seus efeitos colaterais devastadores podem fornecer um "álibi" para elas se sentirem desobrigadas de manter uma atividade sexual compulsória.

No presente estudo notou-se que o relacionamento afetivo-sexual com o parceiro foi referido como satisfatório por cerca da metade das participantes $(49,3 \%)$, enquanto que $50,7 \%$ estavam de algum modo ou totalmente insatisfeitas. $\mathrm{Na}$ análise qualitativa pôde-se notar que, em alguns casos, houve uma melhora momentânea da satisfação com o relacionamento amoroso, creditada a mudanças no comportamento do parceiro, que se mostrou mais próximo e solícito com a mulher durante a fase do tratamento. No entanto, essa mudança, quando houve, foi circunstancial e passageira, culminando posteriormente em decepção, pois, tão logo a mulher completava o tratamento, o parceiro retornava ao padrão anterior de comportamento.

Ainda que a insatisfação seja um traço pregnante no relacionamento afetivo da metade da amostra de mulheres deste estudo, não se observou rompimento da relação marital motivado pelas tensões e atribulações inerentes ao adoecimento e tratamento do câncer. Se, por um lado, esse dado reflete um aspecto positivo, no sentido de que os companheiros não se afastam no período de maior fragilidade das mulheres, por outro lado parece haver certa acomodação de uma parcela expressiva das participantes frente a uma situação relacional vivenciada como insatisfatória.

Outro resultado obtido reforça a hipótese de resignação ante a insatisfação vivenciada com o relacionamento, observada em metade das participantes. A maior parte $(64,3 \%)$ das mulheres que sente que seu relacionamento preenche totalmente suas necessidades afetivas retomou a vida sexual por iniciativa própria (ou de ambos), enquanto que a maioria $(76,5 \%)$ das participantes que mostrou insatisfação retomou as relações sexuais para agradar o parceiro ou por insistência do mesmo. Por meio do componente qualitativo do estudo constatou-se que as razões alegadas pelas mulheres se referem, basicamente, ao afeto que sentem pelo parceiro ou ao sentimento de cumprimento de um "dever conjugal". Esse sentimento está relacionado a uma construção tradicional de gênero, que 
regula a vida sexual da maior parte das participantes deste estudo e que prescreve que a sexualidade feminina deve ser vivida, prioritariamente, como uma obrigação da mulher de gratificar os instintos do companheiro, reforçando assim a subordinação do sexo feminino ao domínio masculino.

Esse resultado evidencia que os fatores associados à dimensão afetiva do relacionamento podem ser tão ou mais decisivos na retomada da atividade sexual do que os efeitos colaterais do tratamento e as alterações psicológicas suscitadas pelo diagnóstico. A literatura tem valorizado mais consistentemente o impacto dos desconfortos físicos sobre o funcionamento sexual da mulher mastectomizada (Archibald Lemieux, Byers, Tamlyn, \& Worth, 2006; Barni \& Mondin, 1997; Cesnik \& Santos, 2012b; Ganz et al., 1998, Ganz et al., 2003; Knobf, 2001; Santos et al., 2013), negligenciando as motivações relacionadas às construções de gênero, que esculpem o relacionamento amoroso e a intimidade entre homens e mulheres engajados em uma relação estável.

Os resultados obtidos neste estudo são consistentes com o encontrado por Brédart et al. (2011), que concluíram que os fatores psicológicos, incluindo a percepção do relacionamento do casal, aparecem com destaque na experiência de problemas sexuais em mulheres sobreviventes ao câncer de mama. A novidade apresentada pelo presente estudo consiste em mostrar que a satisfação com o relacionamento com o parceiro afetivo é um fator intimamente relacionado à saúde sexual após o câncer de mama. Por conseguinte, o profissional de saúde deve prestar atenção também aos aspectos relacionais, como o tipo de vinculação e o padrão de comunicação estabelecidos pelo casal (Rowland et al., 2009), características habitualmente negligenciadas no cuidado. Além disso, para fundamentar o aconselhamento, é preciso conhecer a história reprodutiva e sexual das mulheres em tratamento para o câncer de mama (Vieira et al., 2013). A continuidade, ou interrupção seguida de retomada, da vida sexual provavelmente depende, entre outros aspectos, do padrão anterior de intimidade, comunicação e interação conjugal, em termos de frequência, qualidade e satisfação auferida com o relacionamento afetivo-sexual.

Ao contrário do que se poderia pensar, um número expressivo de mulheres referiu a não interrupção da vida sexual na vigência de seu tratamento. Esse dado corrobora o valor das trocas afetivas com o parceiro como um aspecto fundamental do apoio social recebido durante o tratamento (Ambrósio \& Santos, 2011, 2015; Duarte \& Andrade, 2003). Segundo Manne et al. (2003), muitas pacientes com câncer de mama identificam seus parceiros como sua mais valiosa fonte de apoio durante sua experiência de adoecimento. Considerando que as alterações físicas decorrentes da terapêutica podem acarretar prejuízos na imagem corporal (Fobair et al., 2006; Santos \& Vieira, 2011) e déficits no sentimento de atratividade sexual e feminilidade (Archibald et al., 2006; Barni \& Mondin, 1997; Basson et al., 2004; Brédart et al., 2011; Cesnik \& Santos, 2012a; Speer et al., 2005; Takahashi et al., 2008), o fato de sentir-se aceita pelo companheiro pode oferecer o reasseguramento que a mulher necessita para restaurar sua autoestima abalada. Assim, no presente estudo ficou evidenciado que as características pessoais e relacionais do parceiro íntimo são importantes componentes, considerados pelas mulheres como relacionados não apenas à qualidade da vida sexual, como à retomada (ou mesmo a não interrupção) da vida sexual durante o tratamento. Esse dado é convergente com a literatura, que mostra que a qualidade do relacionamento afetivo afeta a forma como a mulher se adapta ao diagnóstico (Ballantyne, 2004).

Essa hipótese é reforçada pelo resultado que mostra que, entre as mulheres que relataram que interromperam as relações sexuais durante o tratamento, uma parcela expressiva referiu que mantém, atualmente, frequência de três a quatro relações sexuais ao mês. Já aquelas que não interromperam a vida sexual, 57,7\% referiram manter relações acima de cinco vezes por semana. Esse achado sugere que a retomada do relacionamento sexual com o parceiro provavelmente reproduz o padrão anteriormente estabelecido pelo casal. A análise do componente qualitativo do estudo reforça que a frequência sexual é reflexo, entre outros aspectos, da qualidade percebida em relação ao relacionamento, porém não pode ser tomada como sinônimo de qualidade do laço conjugal, uma vez que uma parcela de mulheres refere que se submete às necessidades sexuais instintivas do companheiro como forma de manter a integridade do casamento.

No estudo de Brédart et al. (2011), as mulheres acometidas por câncer de mama, em comparação com as saudáveis, mantinham intercurso sexual com menor frequência semanal e com mais queixas de dispaurenia. Além disso, relataram mais consistentemente ausência ou falta de desejo sexual e maior dificuldade de alcançar o orgasmo. Ainda em relação aos resultados desse estudo francês, observou-se que nas mulheres sexualmente ativas $(71 \%$ das participantes, percentual próximo aos $67,5 \%$ encontrados na amostra do presente estudo), a menor frequência de atividade sexual, diminuição do prazer sexual ou maior desconforto sexual se mostraram associados ao sentimento de distanciamento emocional no casal ou ao medo da relação sexual sentido pelo parceiro, bem como menor nível de funcionamento emocional, idade mais avançada e sintomas como náuseas ou insônia.

A valorização conferida pelas participantes ao respeito e admiração pelo parceiro em um relacionamento, bem como a relevância atribuída à fidelidade conjugal, sobrepujam outros valores, considerados comparativamente menos relevantes, como ter gostos e interesses comuns, contar com a divisão igualitária das tarefas domésticas e uma renda adequada. Como era de se esperar, entre os relatos obtidos no componente qualitativo observou-se que o atributo mais valorizado em um relacionamento amoroso é o afeto, traduzido em termos de respeito e admiração mútuos. Segundo Giddens (2003), as relações amorosas podem ser definidas em termos de amor romântico e de amor confluente. O amor romântico é produto da sociedade do final do século XVIII, caracterizado pela idealização do outro e relacionado à ideia de auto-realização normativamente desejável. $\mathrm{Na}$ relação de amor romântico, o amor sublime suplanta o ardor sexual. O amor romântico foi considerado um domínio feminino, pautado no casamento, monogamia e maternidade, quando se cultiva o ideal do amor eterno. Para Giddens, no século XX a ideia de amor romântico transformou-se no amor confluente e o modelo de relacionamento ideal transmutou do casamento para o relacionamento puro, que pode ser 
entendido por meio da ligação emocional recíproca e contínua entre duas pessoas, cuja duração depende da satisfação de cada um dos parceiros envolvidos. O amor confluente é um amor temporal e contingente, que inclui o erotismo e o deleite tanto para homens como para mulheres, e elimina a moralidade da mulher "respeitável".

Os relatos de uma parcela minoritária das mulheres que participaram do presente estudo refletem as características do amor confluente. Esses dados sugerem que, embora preponderem concepções tradicionalistas, sobretudo nas participantes acima de 50 anos, nota-se que houve, em algumas delas, a incorporação de concepções mais contemporâneas que informam os discursos sobre a sexualidade. Observa-se, nesses casos, uma transição do ideal de relacionamento, que aos poucos modifica as concepções do modelo tradicional e dá lugar ao modelo do relacionamento igualitário.

\section{Considerações Finais}

A interrupção e retomada da vida sexual após o adoecimento pelo câncer de mama estão intimamente relacionados a aspectos importantes da sexualidade percebidos pelas mulheres, que extrapolam o impacto psicológico do diagnóstico e os efeitos adversos decorrentes dos tratamentos a que são submetidas. Tais aspectos incluem as concepções pessoais de sexualidade, influenciadas pelas relações de gênero e baseadas no cenário cultural, que atuam como reguladoras da conduta sexual, e a qualidade do relacionamento estabelecido com o parceiro. Essa constatação se evidencia a partir da articulação dos resultados obtidos com mulheres que interromperam definitivamente a vida sexual após a doença, com aquelas que recomeçaram as atividades sexuais após algum tempo e, ainda, com aquelas que se mantiveram sexualmente ativas durante todo o processo de diagnóstico e de tratamento.

Esta pesquisa evidencia a importância de se investigarem, em estudos futuros, aspectos relacionados aos valores atribuídos à sexualidade e ao relacionamento afetivo-sexual, quando se estudam os fatores associados à ausência ou a menor frequência de atividade sexual, diminuição do prazer sexual, elevado desconforto ou insatisfação sexual em decorrência do tratamento oncológico.

Em relação às implicações para a prática, os achados trazem contribuições valiosas para os profissionais que atuam na área da oncologia, uma vez que a saúde sexual tem sido considerada elemento central para assegurar o bem-estar psicológico e a qualidade de vida. Com efeito, a intimidade sexual tem sido valorizada como uma dimensão crucial para que a dolorosa experiência do tratamento do câncer de mama possa ser mais tolerável e menos difícil de ser manejada. A identificação de necessidades relacionadas a essa dimensão pode contribuir para prover a assistência apropriada durante o processo de reabilitação psicossocial, auxiliando a mulher a restaurar sua autoestima e a fortalecer seu sentimento de esperança na possibilidade de superação, mediante o uso de estratégias de enfrentamento adaptativas.

\section{Referências}

Ambrósio, D. C. M., \& Santos, M. A. (2011). Vivências de familiares de mulheres com câncer de mama: uma compreensão fenomenológica. Psicologia: Teoria e Pesquisa, 27(4), 475484.

Ambrósio, D. C. M., \& Santos, M. A. (2015). Apoio social à mulher mastectomizada: um estudo de revisão. Ciência \& Saúde Coletiva, 20(3), 851-864. doi: https://dx.doi.org/10.1590/141381232015203.13482014

Archibald, S., Lemieux, S., Byers, E. S., Tamlyn, K., \& Worth, J. (2006). Chemically-induced menopause and the sexual functioning of breast cancer survivors. Women \& Therapy, 29(1-2), 83-106.

Ballantyne, P. J. (1999). The social determinants of health: A contribution to the analysis of gender differences in health and illness. Scandinavian Journal of Public Health, 27, 290-295.

Ballantyne, P. J. (2004). Social context and outcomes for the ageing breast cancer patient: considerations for clinical practitioners. Journal of Clinical Nursing, 13(3a), 11-21.

Barni, S., \& Mondin, R. (1997). Sexual dysfunction in treated breast cancer patients. Annals of Oncology, 8(2), 149-153.

Basson, R., Leiblum, S., Brotto, L., Derogatis, L., Fourcroy, J., Fugl-Meyer, K., ... Schultz, W. W. (2004). Revised definitions of women's sexual dysfunction. Journal of Sexual Medicine, 1(1), 40-48.

Brédart, A., Dolbeault, S., Savignoni, A., Besancenet, C., This, P., Giami, A., ... ... Copel, L. (2011). Prevalence and associated factors of sexual problems after early-stage breast cancer treatment: results of a French exploratory survey. PsychoOncology, 20(8), 841-850.

Cesnik, V. M., \& Santos, M. A. (2012a). Mastectomia e sexualidade: Uma revisão integrativa. Psicologia: Reflexão e Crítica, 25(2), 339-349.

Cesnik, V. M., \& Santos, M. A. (2012b). Do the physical discomforts from breast cancer treatments affect the sexuality of women who underwent mastectomy?. Revista da Escola de Enfermagem da USP, 46(4), 1001-1008.

Cesnik, V. M., Vieira, E. M., Giami, A., Almeida, A. M., Santos, D. B., \& Santos, M. A. (2013). The sexual life of women with breast cancer: Meanings attributed to the diagnosis and its impact on sexuality. Estudos de Psicologia (Campinas), 30(2), 187-197.

Creswell, J. W., \& Clark, V. L. (2011). Designing and conducting mixed methods research. London: Sage Publications.

Denzin, N. K. (2009). The research act: A theoretical introduction to sociological methods. New Brunswick, NJ: Aldine Transaction.

Duarte, T. P., \& Andrade, A. N. (2003). Enfrentando a mastectomia: análise dos relatos de mulheres mastectomizadas sobre questões ligadas à sexualidade. Estudos de Psicologia (Natal), $8(1), 155-163$.

Fobair, P., Stewart, S. L., Chang, S. B., D’Onofrio, C., Banks, P. J., \& Bloom, J. R. (2006). Body image and sexual problems in young women with breast cancer. Psycho-Oncology, 15(7), 579-594.

Ganz, P. A., Desmond, K. A., Belin, T. R., Meyerowitz, B. E., \& Rowland, J. H. (1999). Predictors of sexual health in women after a breast cancer diagnosis. Journal of Clinical Oncology, 17(8), 2371-2371. 
Ganz, P. A., Desmond, K. A., Leedham, B., Rowland, J. H., Meyerowitz, B. E., \& Belin, T. R. (2002). Quality of life in long-term, disease-free survivors of breast cancer: A follow-up study. Journal of the National Cancer Institute, 94(1), 39-49.

Ganz, P. A., Greendale, G. A., Petersen, L., Kahn, B., \& Bower, J. E. (2003). Breast cancer in younger women: Reproductive and late health effects of treatment. Journal of Clinical Oncology, 21(22), 4184-4193.

Ganz, P. A., Rowland, J. H., Desmond, K., Meyerowitz, B. E., \& Wyatt, G. E. (1998). Life after breast cancer: Understanding women's health-related quality of life and sexual functioning. Journal of Clinical Oncology, 16(2), 501-514.

Giddens, A. (2003). A transformação da intimidade: sexualidade, amor e erotismo nas sociedades modernas (M. Lopes, Trans.). São Paulo: UNESP.

Janz, N. K., Mujahid, M., Lantz, P. M., Fagerlin, A., Salem, B., Morrow, M., Deapen, D., \& Katz, S. J. (2005). Population-based study of the relationship of treatment and sociodemographics on quality of life for early stage breast cancer. Quality of Life Research, 14(6), 1467-1479.

Klaeson, K., Sandell, K., \& Bertero, C. M. (2011). To feel like an outsider: Focus group discussions regarding the influence on sexuality caused by breast cancer treatment. European Journal of Cancer Care, 20(6), 728-737.

Knobf, M. T. (2001). The menopausal symptom experience in young mid-life women with breast cancer. Cancer Nursing, 24(3), 201-211.

Lund, T. (2012). Combining qualitative and quantitative approaches: some arguments for mixed methods research. Scandinavian Journal of Educational Research, 56(2), 155-165.

Manne, S., Ostroff, J., Sherman, M., Glassman, M., Ross, S., Goldstein, L., \& Fox, K. (2003). Buffering effects of family and friend support on associations between partner unsupportive behaviors and coping among women with breast cancer. Journal of Social and Personal Relationships, 20(6), 771-792.

Meyerowitz, B. E., Desmond, K. A., Rowland, J. H., Wyatt, G. E., \& Ganz, P. A. (1999). Sexuality following breast cancer. Journal of Sex and Marital Research, 25(3), 237-250.

Peres, R. S., \& Santos, M. A. (2007). Breast cancer, poverty and mental health: emotional response to the disease in women from popular classes. Revista Latino-Americana de Enfermagem, 15(Esp.), 786-791.

Peres, R. S., \& Santos, M. A. (2009). Personalidade e câncer de mama: Produção científica em psico-oncologia. Psicologia: Teoria e Pesquisa, 25(4), 611-620.

Peres, R. S., \& Santos, M. A. (2012). Psicossomática psicanalítica: Intersecções entre teoria, pesquisa e clínica. Campinas, SP: Alínea.

Rowland, J. H., Meyerowitz, B. E., Crespi, C. M., Leedham, B., Desmond, K., Belin, T. R., \& Ganz, P. A. (2009). Addressing intimacy and partner communication after breast cancer: A randomized controlled group intervention. Breast Cancer Research and Treatment, 118(1), 99-111.

Santos, D. B., \& Vieira, E. M. (2011). Imagem corporal de mulheres com câncer de mama: Uma revisão sistemática da literatura. Ciência \& Saúde Coletiva, 16(5), 2511-2522.
Santos, D. B., Ford, N. J., Santos, M., \& Vieira, E. M. (2014). Breast cancer and sexuality: The impacts of breast cancer treatment on the sex lives of women in Brazil. Culture, Health \& Sexuality: An International Journal for Research, Intervention and Care, 16(3), 246-257.

Santos, D. B., Santos, M. A., \& Vieira, E. M. (2014). Sexualidade e câncer de mama: Uma revisão sistemática da literatura. Saúde \& Sociedade, 23(4), 1342-1355.

Santos, M. A., Peres, R. S., Ferreira, S. M. A., Gozzo, T. O., Panobianco, M. S., Almeida, A. M. (2013). A (in)sustentável leveza dos vínculos afetivos: Investigando a sexualidade em mulheres que enfrentam o tratamento do câncer de mama. Vinculo: Revista do NESME, 10(1), 1-8.

Sawin, E. M. (2012). “The body gives way, things happen”: older women describe breast cancer with a non-supportive intimate partner. European Journal of Oncology Nursing, 16(1), 64-70.

Scorsolini-Comin, F., Santos, M. A., \& Souza, L. V. (2009). Vivências e discursos de mulheres mastectomizadas: Negociações e desafios do câncer de mama. Estudos de Psicologia (Natal), 14(1), 41-50.

Silva, G., \& Santos, M. A. (2008). "Será que não vai acabar nunca?”: Perscrutando o universo do pós-tratamento do câncer de mama. Texto \& Contexto - Enfermagem, 17(3), 561-568.

Silva, G., \& Santos, M. A. (2010). Stressors in breast cancer posttreatment: A qualitative approach. Revista Latino-Americana de Enfermagem, 18(4), 688-695.

Speer, J. J., Hillenberg, B., Sugrue, D. P., Blacker, C., Kresge, C. L., Decker, V. B., ... Decker, D. A. (2005). Study of sexual functioning determinants in breast cancer survivors. The Breast Journal, 11(6), 440-447.

Takahashi, M., Ohno, S., Inoue, H., Kataoka, A., Yamaguchi, H., Uchida, Y., ... Kai, I. (2008). Impact of breast cancer diagnosis and treatment on women's sexuality: A survey of Japanese patients. Psycho-Oncology, 17(9), 901-907.

Ussher, J. M., Perz, J., \& Gilbert, E. (2012). Changes to sexual well-being and intimacy after breast cancer. Cancer Nursing, 35(6), 456-465.

Ussher, J. M., Perz, J., \& Gilbert, E. (2013). Information needs associated with changes to sexual well-being after breast cancer. Journal of Advanced Nursing, 69(2), 327-337.

Vieira, E. M., Santos, D. B., Santos, M. A., \& Giami, A. (2014). Experience of sexuality after breast cancer: A qualitative study with women in rehabilitation. Revista Latino-Americana de Enfermagem, 22(3), 408-414.

Vieira, E. M., Yoshinari Júnior, G. H., Souza, H. C. C., Mancini, M. P. M., \& Perdoná, G. S. C. (2013). História reprodutiva e sexual de mulheres tratadas de câncer de mama. Revista Brasileira de Ginecologia e Obstetrícia, 35(2), 78-83.

Recebido em 16.01.2014

Primeira decisão editorial em 28.02.2016

Versão final em 25.04.2016

Aceito em 25.04.2016 
